\title{
A Novel Magnetic Coupling Configuration for Enhancing the Torque Density
}

\author{
Yusuf Akcay ${ }^{1}$, Paolo Giangrande ${ }^{1}$, Michael Galea ${ }^{1,2}$ \\ ${ }^{1}$ PEMC Research Group, University of Nottingham, Nottingham, United Kingdom \\ ${ }^{2}$ Key Laboratory of More Electric Aircraft Technology of Zhejiang Province, University of Nottingham \\ Ningbo China \\ Email: yusuf.akcay1@nottingham.ac.uk
}

\begin{abstract}
This paper introduces a novel magnetic coupling (MC) topology, which is able to achieve significant torque density values by adding a limited amount of extra permanent magnets (PMs). Indeed, the proposed MC architecture, namely enhanced magnetic coupling (EMC), includes both axial and radial concepts in the same structure. In particular, one radial and two axial MCs are considered and embedded in a single device. Hence, this configuration allows to boost the transmitted torque that is sum of three contributions, while keeping almost unaltered the overall device's volume. The geometry of the EMC is discussed and a 2D subdomain analysis is employed for its preliminary study and fast performance evaluation. The results obtained by the 2D subdomain approach are then compared and validated against 3D finite element method (FEM).
\end{abstract}

\section{INTRODUCTION}

The MCs transfer torque/speed from a prime mover to a load by exploiting the magnetic interaction between the PMs without any physical contact. This solution enables maintenance-free and reliable torque/speed transmission over the mechanical counterparts [1]-[2]. Although the wellestablished know-how on traditional mechanical couplings, MCs can be found in several industrial applications. Further, they represent a suitable solution for isolated systems, where the mechanical power needs to be transmitted across a separation wall. In terms of architecture, radial and axial MCs are the most common configurations and their prominent advantages are high efficiency (i.e. very low mechanical losses) and absence of mechanical wear-out of the moving parts. However, innovative MC's architectures are being proposed to meet the application constraints and improve the overall performance. For instance, a tubular type MC is introduced and analyzed in [3], which has been conceived for a linear application. Current research is also focused on improving the torque transmission capability of the MCs, since their torque density value is lower than that featured by the mechanical devices [4]. Indeed, a higher torque density leads to a more compact design [5], which allows to comply with the stringent available space typically demanded in transportation electrification fields [6]-[8].

In this prospective, a new MC concept is proposed in the present work, which combines both radial and axial architectures in a single device with the purpose of boosting the overall torque density. In fact, the novel EMC ensures a higher developed torque with the same or even less PM's volume compared to that required by a radial MC assumed as benchmark for the comparative analysis [9]. The investigated EMC includes one radial $\mathrm{MC}$ and two axial $\mathrm{MCs}$, as shown in Fig.1. Considering the two axial MCs, their PMs are located on the lateral edges of the radial MC aiming at maximizing the volume exploitation.

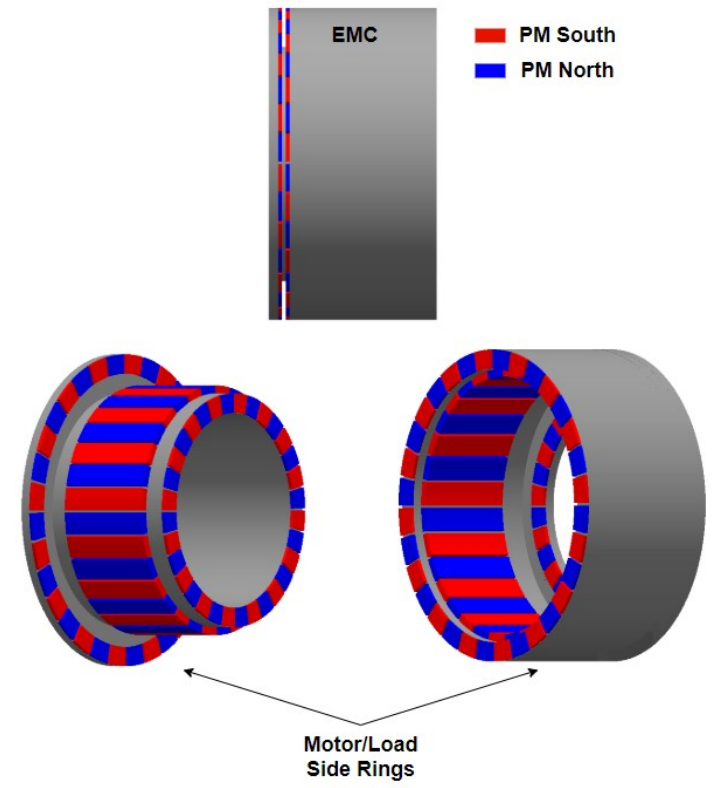

Fig. 1. Layout of the proposed EMC.

The EMC's performance analysis has been carried out through a 2D analytical subdomain method, which separately determines the solution for each MC constituting the EMC. Such analytical approach has been used to assess and identify the most important design parameters (i.e. airgap thickness values, pole-pair numbers, etc.) that mainly affect the torque density of the EMC [10]. The results have also been verified via $3 \mathrm{D}$ FEM that allows to account for end effects [11]. Finally, a comparative exercise between EMC and benchmark radial MC has been addressed and the corresponding outcomes have been discussed.

\section{THE PROPOSED EMC}

MCs are used for contactless mechanical power transmission due to magnetic fields established between PMs. Thus, mechanical wear-out is avoided reducing the maintenance time and increasing the reliability level, as common required by modern applications [12]-[14]. Both axial and radial configurations work based on the same principle. However, depending on the application requirements, a suitable configuration is chosen for the system. The proposed configuration exhibits both axial and radial MCs in a single pattern aiming to boost torque density by increasing the PM interaction region. This section describes the proposed coupling structure and identifies the size parameters for the analytical and FEM evaluations. The section continues to explain the 2D analytical method and lastly gives comparative analysis with 3D FEM for a preliminary assessment. 


\section{A. Coupling Structure}

The overall 3D layout of the EMC is illustrated in Fig. 1, while the half $2 \mathrm{D}$ cross section representation is given in Fig. 2, for the sake of clarity. The geometrical parameters covering both sections are listed in Table I. It should be noted that the parameters are for only a preliminary assessment and geometrical identification. The analysis related to the most effective parameters, such as pole-pair numbers, PM lengths, are provided in the following sections.

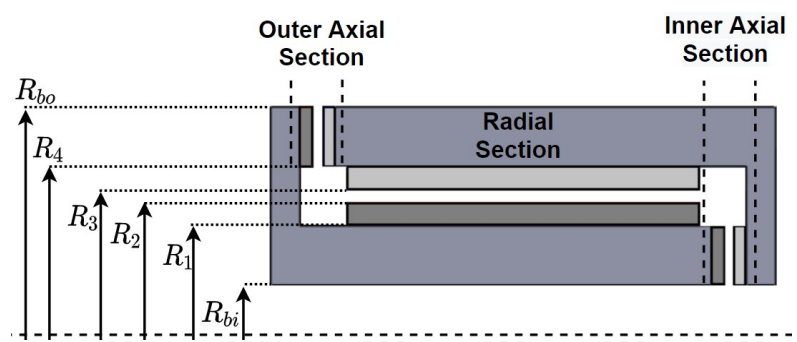

Fig. 2. 2D overview of EMC with details of axial and radial parts.

TABLE I $k N m / m^{3}$

THE EMC PARAMETERS FOR THE PRELIMINARY EVALUATION

\begin{tabular}{ccc}
\hline \hline Symbol & Parameters & Values \\
\hline$R_{b i}$ & Inner Back Iron Inner Radius & $50 \mathrm{~mm}$ \\
$R_{1}$ & Inner PM Inner Radius & $60 \mathrm{~mm}$ \\
$R_{2}$ & Inner PM Outer Radius & $64 \mathrm{~mm}$ \\
$R_{3}$ & Outer PM Inner Radius & $66 \mathrm{~mm}$ \\
$R_{4}$ & Outer PM Outer Radius & $70 \mathrm{~mm}$ \\
$R_{b o}$ & Outer Back Iron Outer Radius & $80 \mathrm{~mm}$ \\
$l_{a}$ & Airgap Thickness & $2 \mathrm{~mm}$ \\
$p$ & PM pole-pair number & 16 \\
$l_{m}$ & PM Thickness & $4 \mathrm{~mm}$ \\
$l_{b a}$ & Back Iron Length, Axial Sections & $5 \mathrm{~mm}$ \\
$l_{s}$ & Total Active Length & $87 \mathrm{~mm}$ \\
$B_{m}$ & PM Openings & $95 \%$ \\
$B_{r}$ & Remanence of PMs & $1.29 \mathrm{~T}$ \\
\hline
\end{tabular}

\section{B. Analytical Implementation}

The 2D analytical methods presented in this paper uses the summation of the torque values determined for the axial and radial sections. Indeed, the adopted analytical approach consists in two separate evaluations, each considering a single sub-system (i.e. axial or radial section of the EMC architecture). The method used for the axial sections, detailed in [15], simplifies the analysis by taking a cylindrical cutting surface at the mean radius of the PMs on the inner and outer axial sections. The 2D model of the axial sections at the mean radius of the PMs is reported in Fig. 3a, whereas the radial section is formulated in $2 \mathrm{D}$ polar coordinate to describe the problem. Both the 2D analytical methods used in this paper for axial and radial sections are based on the resolution of Laplace's and Poisson's equations for the airgap and PM regions, respectively. The detailed solution to procure the general equations of potential functions can be found in [15] for the axial sections.

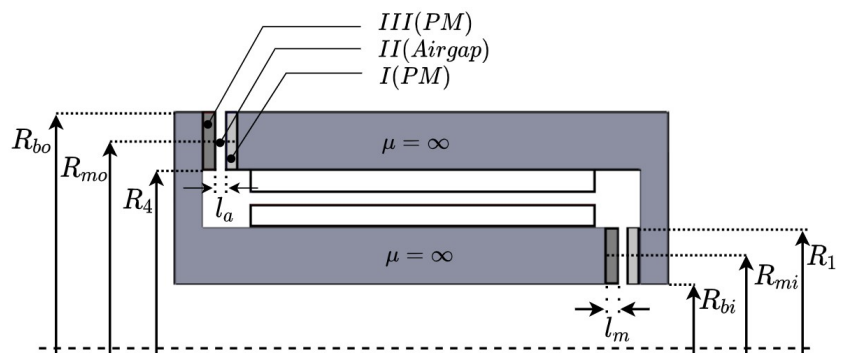

(a)

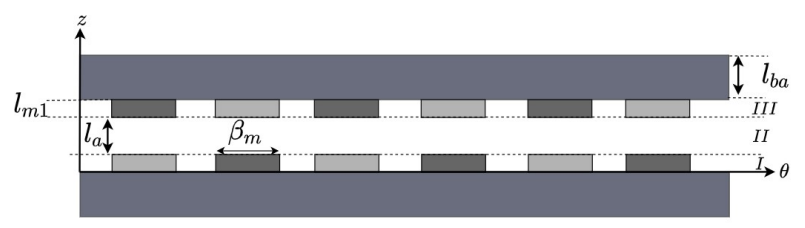

(b)

Fig. 3. Linearized 2D representation of axial sections; (a) frontal view, (b) lateral view.

Airgap and PM subdomains have their magnetic vector potential varying with $\theta$ and $\mathrm{z}$ coordinates as highlighted in Fig. $3 \mathrm{~b}$. Using the derivative of the potential equation in the airgap subdomain, the radial and tangential components of the flux density distribution can be found as described in (1) and (2).

$$
\begin{gathered}
B_{I I z}=-\frac{1}{R_{m o, m i}} \frac{\partial A_{I I}}{\partial \theta} \\
B_{I I \theta}=\frac{\partial A_{I I}}{\partial z}
\end{gathered}
$$

where $A_{I I}$ is the vector potential in the airgap subdomain. Thus, the torque expression for the inner and outer axial sections is processed according to the Maxwell stress tensor as in (3). The angle $\zeta$ represents the shift between both sides of PMs.

$$
T_{e}=\frac{R_{1, b o}^{3}-R_{b i, 4}^{3}}{3 \mu_{0}} \int_{0}^{2 \pi} B_{I I \theta}(\theta, \zeta) B_{I I z}(\theta, \zeta) d \theta
$$

The radial section follows similar steps for the development of the analytical method. The general equations for the magnetic vector potential in each region are proceeded by using the Laplace's and Poisson's equations in polar coordinate. The PM in the radial section is assumed radially magnetized, while the parallel and Halbach magnetization are analyzed in [16]. For the radial section, the subdomains are shown in Fig. 4, along with the corresponding parameters.

The assumptions adopted for the $2 \mathrm{D}$ analytical method representation of the EMC are:

- Permeability of the iron parts is infinite.

- Relative recoil permeability of PMs is 1 . 


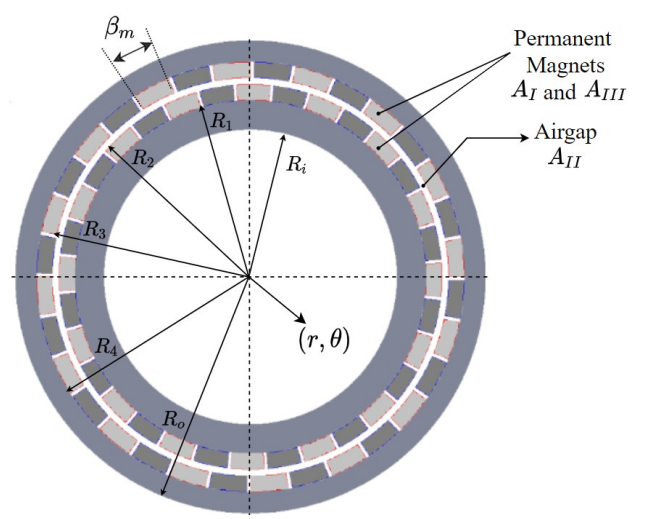

Fig. 4. Overview of the radial section for the 2D analytical method.

The flux density components are given in (4) and (5).

$$
\begin{aligned}
& B_{I I r}(r, \theta)=\frac{1}{r} \frac{\partial A_{I I}}{\partial \theta} \\
& B_{I I \theta}(r, \theta)=-\frac{\partial A_{I I}}{\partial r}
\end{aligned}
$$

Eq. (4) and (5) are substituted in (6) in order to obtain the electromagnetic torque values. The air-gap subdomain with a specified radius is used over the integral path for the torque expression according to (6).

$$
T_{e}=\frac{L R_{e}^{2}}{2 \mu_{0}} \int_{0}^{2 \pi} B_{I I r}\left(R_{e}, \theta\right) B_{I I \theta}\left(R_{e}, \theta\right) d \theta
$$

where $R_{e}$ represents the average airgap radius and $\theta$ is the angle of the radial and tangential components of the flux density distribution.

\section{Flux Density and Torque Calculations}

In this section, the existing 2D analytical methods for the axial and radial sections are implemented by using the parameters provided in Table I. For a validation purpose, the results delivered by the $2 \mathrm{D}$ analytical approach are compared against those related to a 3D FEM. The 3D analysis is performed through the simulation software MagNet [17] on the geometry defined by Table I, while the mesh size distribution of the 3D FEM is depicted in Fig. 5a. Considering a rotational speed of $5000 \mathrm{rpm}$ at no-load condition meaning that there is no torque angle between motor and load side, the flux density distribution for the EMC is preliminary evaluated, as shown in Fig. 5b.

The first prominent comparison is elaborated by giving the flux density distributions of all sub-systems under the full-load condition ( $\zeta=5.625^{\circ}$ ), i.e. the inner and outer axial, and radial. Focusing on the only radial sub-system, the axial and tangential components of the flux density distributions within the airgap are reported in Fig. 6 for both the $2 \mathrm{D}$ analytical method and 3D FEM. The results of Fig. 6 reveal a good agreement between $2 \mathrm{D}$ analytical and $3 \mathrm{D}$ FEM approaches. Conversely, the same comparative exercise has been repeated for the two axial subsystems (i.e. outer and inner axial sections) and the trends of the flux density components inside the airgap are given in Fig. 7 and 8 , respectively. From these figures, it is possible to note that the radial component of the flux density distribution is underestimated by the analytical method. This might be due by the intrinsic approximation typical of the analytical approach.
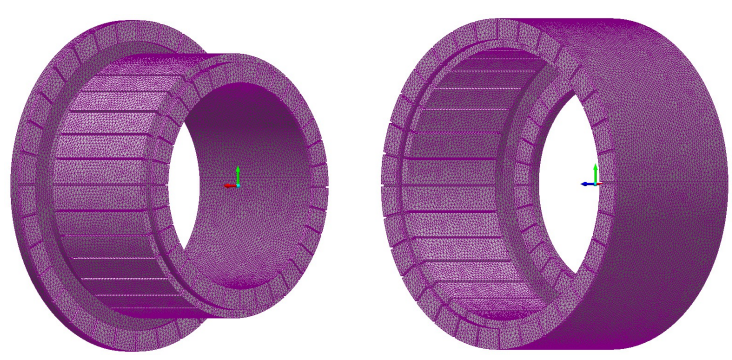

(a)
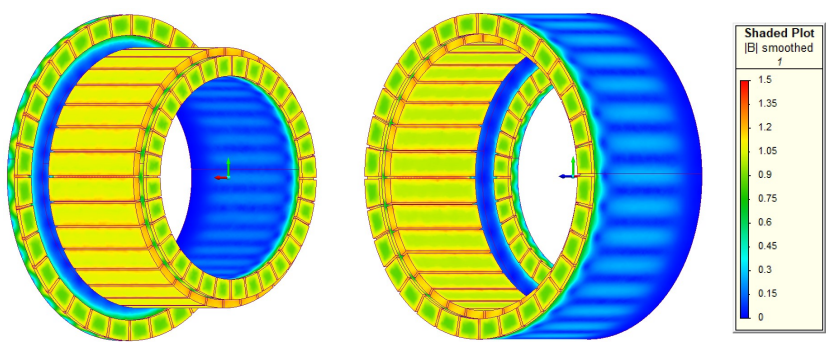

(b)

Fig. 5. The mesh size distribution (a), and the flux density distribution (b) of the EMC under no-load condition.

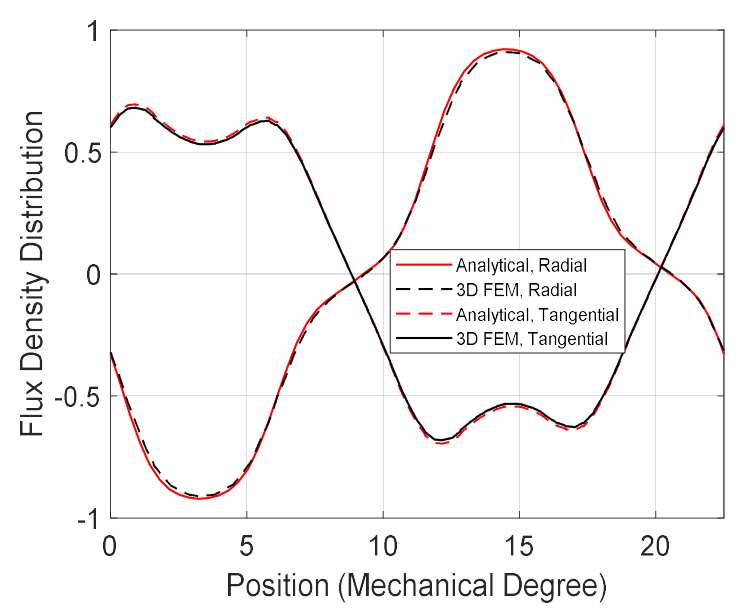

Fig. 6. Analytical and FEM comparison of the flux density distribution in the airgap of the radial section at the maximum torque point.

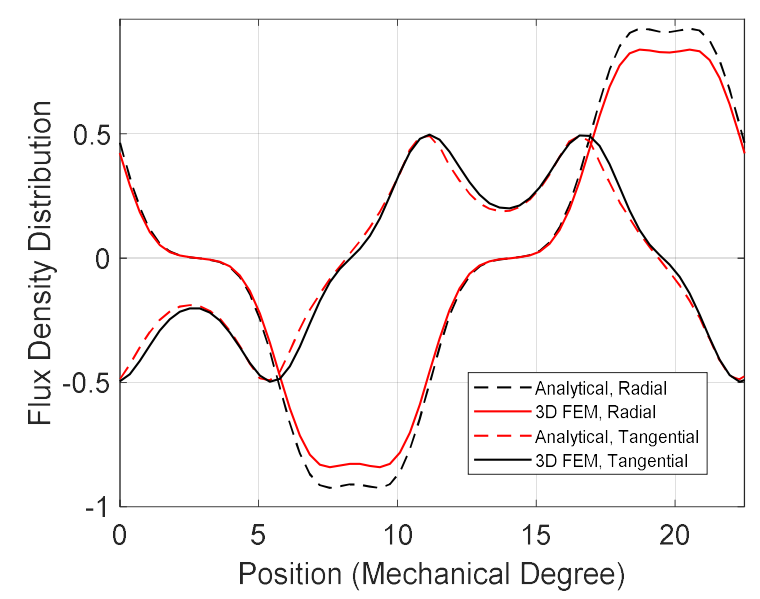

Fig. 7. Analytical and FEM comparison of the flux density distribution in the airgap of the outer axial section at the maximum torque point. 


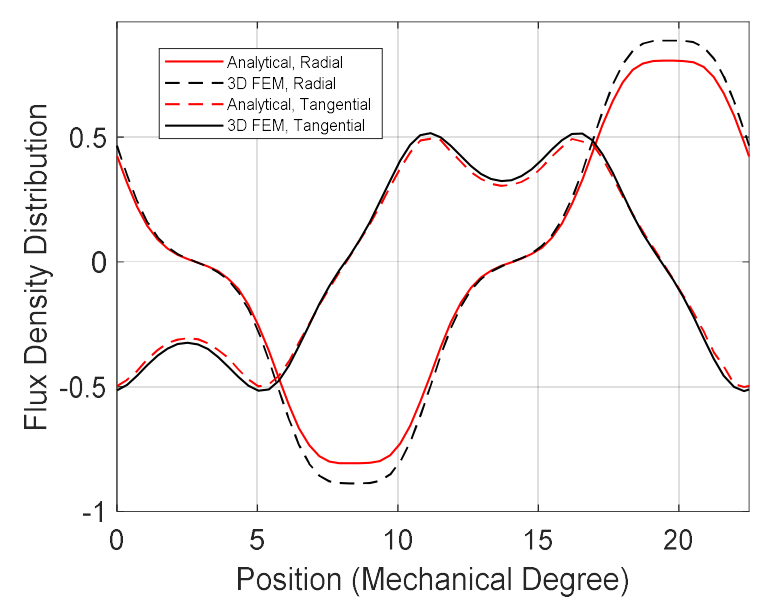

Fig. 8. Analytical and FEM comparison of the flux density distribution in the airgap of the inner axial section at the maximum torque point.

Determined the flux density components, the overall electromagnetic torque transmitted by the EMC is computed by adding the torque terms corresponding to each of the subsystems (i.e. radial, outer and inner axial) composing the EMC. It is important to mention that the pole-pair numbers are kept the same for all sections. Thus, the static torque of each section is supplied within the same period. An interesting approach indicated in [18] could be implemented in the EMC depending on application requirements where a pulsatile torque is needed. Fig. 9 gives the static torque values separately for each section, whilst the net torque is plotted in Fig. 10. In these figures, the static torque is considered over a mechanical angle of 11.25 degrees (i.e. half cycle of the static torque) and its the peak occurs at 5.625 degrees.

The 2D analytical method exhibits a set of limitations as pointed out in the previous section. In fact, the analytical methods used for the axial and radial sections determine the potential functions based on the 2D simplified geometries. However, more accurate results can be achieved by introducing a correction factor, as discussed in [19], where sinusoidal trend of the static torque is exploited. Nevertheless, the implemented analytical tool permits a quick evaluation of the EMC torque density, while several design parameters could be varied in a wide range, as often requires at the early design stage [20]. In the next section, the introduced 2D analytical model is used as preliminary design tool to assess the performance of the proposed EMC, which are then compared to size-compatible standard radial MC.

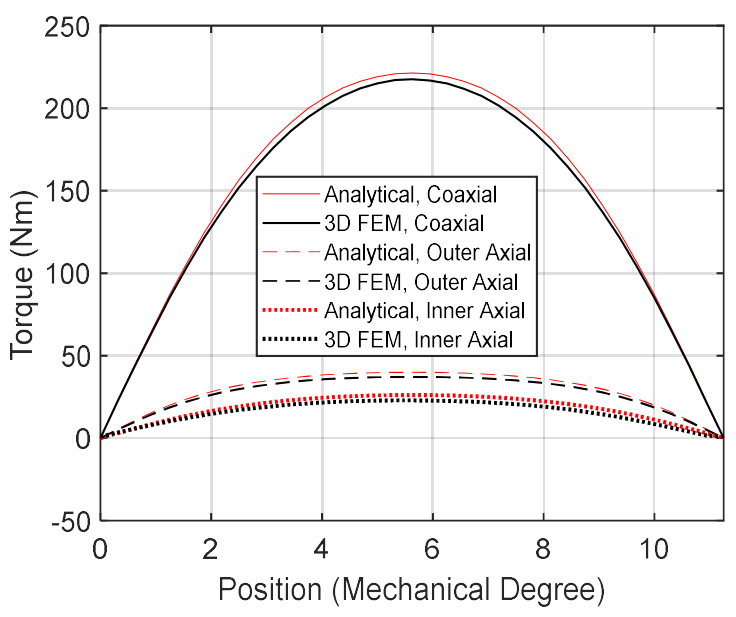

Fig. 9. The static torque comparison of each sections, i.e. axial and radial.

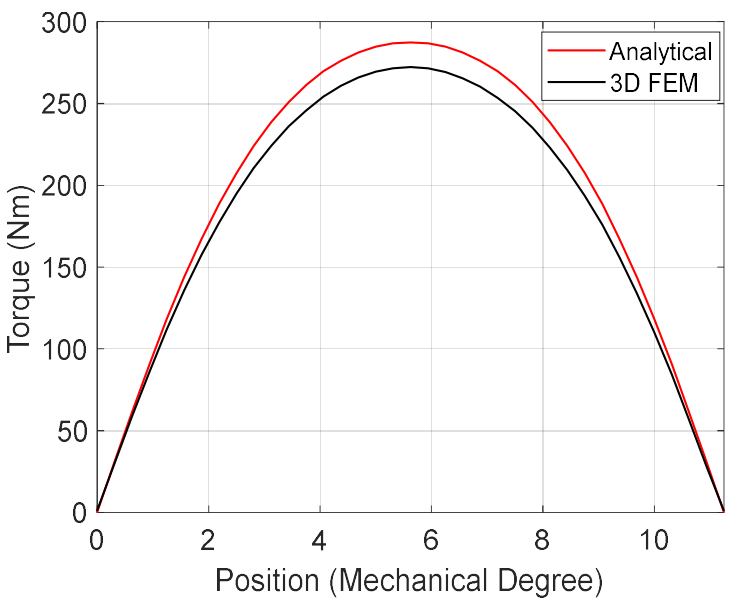

Fig. 10. Overall EMC static torque comparison between the $2 \mathrm{D}$ analytical methods and 3D FEM.

\section{RESULTS AND DISCUSSION}

Since the effectiveness of the 2D analytical method has been proved against 3D FEM, in this section competitive studies between EMC and radial MC. The first study (i.e. 2D analytical analysis) aims to observe the effects of the airgap thickness and pole-pair numbers on torque density. According to the outcomes of the first study, the best performing pole-pairs number is then selected and kept for the later study. During the second study (i.e. 3D FEM analysis), the torque density trend as function of the volume is evaluate for both EMC and radial MC using 3D FEM. The outcome of the second study are separately presented considering in turn the active part volume and PM volume for computing the torque density (i.e. volumetric torque density).

\section{A. 2D Analytical Analysis}

The comparative study presented here relies on the listed in Table II. It should be noted that the radius $R_{1}$ is chosen as a reference-start-point to build both the EMC and radial MC. Thus, the radial section of the EMC and radial MC possess the same size and volume for a fair comparison. However, since the radial MC does not require the same back-iron thickness, its value is less in the outer and inner part than the EMC counterpart. It can be seen in Table II that the outer ring radius is $75 \mathrm{~mm}$ in the EMC, whereas it is $68 \mathrm{~mm}$ in the radial $\mathrm{MC}$.

TABLE II

2D ANALYTICAL ANALYSIS CONSTRAINS

\begin{tabular}{ccc}
\hline \hline Parameter & EMC & CMC \\
\hline PM Thickness (mm), $l_{m}$ & 5 & 5 \\
Inner Ring Radius (mm), $R_{b i}$ & 35 & 40 \\
Outer Ring Radius (mm), $R_{b o}$ & 75 & 68 \\
Back Iron Thickness, Axial Sections, $l_{b a}$ & 5 & - \\
Remanence of the PMs, $B_{r}$ & $1.29 \mathrm{~T}$ & $1.29 \mathrm{~T}$ \\
Airgap Thickness, $l_{a}$ & Variable & Variable \\
PM pole-pair number, $p$ & Variable & Variable \\
PM Thickness, $l_{m}$ & $5 \mathrm{~mm}$ & $5 \mathrm{~mm}$ \\
Back Iron Length, Axial Sections, $l_{b a}$ & $5 \mathrm{~mm}$ & $5 \mathrm{~mm}$ \\
PM Openings, $B_{m}$ & $95 \%$ & $95 \%$ \\
Remanence of PMs, $B_{r}$ & $1.29 \mathrm{~T}$ & $1.29 \mathrm{~T}$ \\
\hline
\end{tabular}


Based on the above considerations, the torque density maps of the EMC and radial MC are reported in Fig. 11 and 12 , respectively. In particular, the torque density trend is expressed as function of the airgap thickness, which varies between $0.1 \mathrm{~mm}$ and $2 \mathrm{~mm}$, and the pole-pair number, which changes in the range between 2 and 50. For the proposed EMC, the same value of the pole-pair number is considered for each subsystems (i.e. radial, outer and inner axial ) composing the EMC. Further, the determined torque density values are referred to the active part volume.

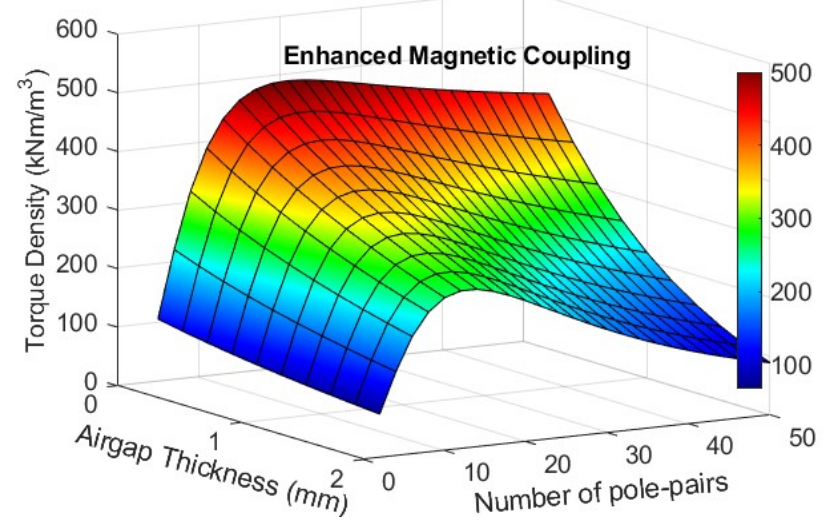

Fig. 11. Torque density trend for the EMC with different pole-pairs number and airgap thickness (analytical analysis).



Fig. 12. Torque density trend for the radial MC with different pole-pairs number and airgap thickness (analytical analysis).

Relying on the sensitivity analysis of Fig. 11 and 12, the most suitable pole-pair number is equal to 12 with $2 \mathrm{~mm}$ airgap thickness. In general, the torque density comparison gives promising results for the EMC.

\section{B. 3D FEM Analysis}

According to the results obtained in the previous section, the best torque density is reached with the pole-pair number of 12 and an airgap thickness of $2 \mathrm{~mm}$.

The 3D finite element models have been built for both EMC and radial MC. The radial section of the EMC and the radial $\mathrm{MC}$ has almost identical size parameters. However, since the EMC exhibits the axial sections on the sides, the back-iron thickness has different geometry than the radial MC. Thus, in order to ensure a fair comparison, the models are built with a reference point where the radial part PMs are placed in the same radius in both topologies. It should also be noted that the active length of the radial $\mathrm{CMC}$ is considered the PM length. There is not additional section placed on the end of the PMs. In contrast, the EMC holds a safe distance between the radial section and axial section PMs to build a safe electromagnetic circuit path in the back iron. For that reason, the active length of the EMC includes the back-iron thickness of both inner and outer axial sections.

In this section, the torque density trend has been determined for different inner ring radius, when pole-pair number, airgap thickness and PM thickness are kept constant. In Fig. 13, the trend of the torque density calculated considering the active part volume is shown, as function of the inner ring radius. Similarly, the torque density computed according to the PM's volume is also provided in Fig. 14. These figures compare 2D analytical and 3D FEM outcomes for both EMC and radial MC. The obtained results clearly indicate the torque density improvement by adopting the proposed EMC. It is important to note that the torque density can be increased significantly while having the same or less PM volume in the EMC.

In addition, the EMC can further be improved depending on applications requirements. In [21], the study shows that the electromagnetic torque changes by changing the polepair number and the overall diameter in both axial and radial topologies. Thus, the EMC can exhibit different pole-pair numbers in order to optimize the net torque. However, as mentioned in [18], changing pole numbers in different parts would lead non-sinusoidal torque cycles.

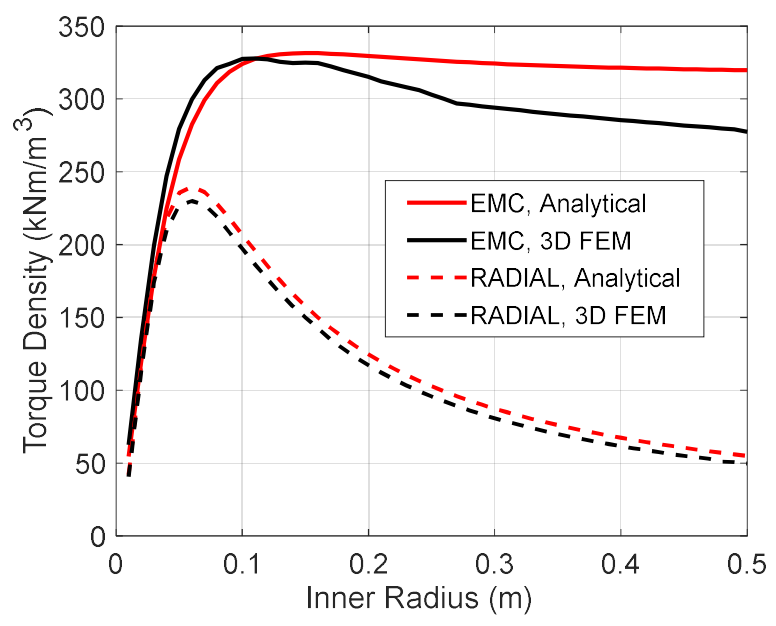

Fig. 13. Torque density comparison of the EMC and radial MC considering the active part volume.

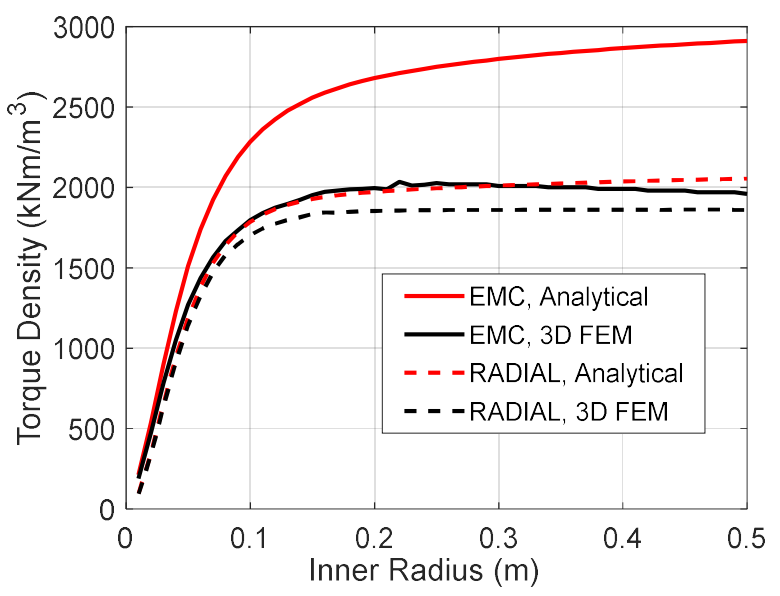

Fig. 14. Torque density comparison between the EMC and radial MC considering only PM volume. 


\section{CONCLUSION}

In this paper, a novel magnetic coupling topology has been presented and analyzed. The proposed EMC embeds both axial and radial coupling architectures in single device. The principle operation and torque transmission capability have been analyzed by the 2D analytical methods and 3D FEM. The analytical methods allow quick evaluation of wide set of parameters and gives relatively good matchings compared to $3 \mathrm{D}$ FEM. It has been observed that the proposed EMC achieves promising torque density compared with the commonly used radial MC. The EMC delivers 270 $\mathrm{kNm} / \mathrm{m}^{3}$ torque density value, which is 11 percent more than the radial $\mathrm{MC}$, at 12 pole pairs and $2 \mathrm{~mm}$ airgap thickness. In addition, the later analysis with 3D FEM comparison proves that the proposed EMC has significantly better torque density depending on the radius with the same or less PM volume.

\section{ACKNOWLEDGMENT}

This project received funding from the Clean Sky 2 Joint Undertaking under the European Union's Horizon 2020 research and innovation programme under grant agreements no 821023 and no. 807081. This work was also partially funded by the University of Nottingham Propulsion Futures Beacon.

\section{REFERENCES}

[1] J. -. Yonnet, S. Hemmerlin, E. Rulliere and G. Lemarquand, "Analytical calculation of permanent magnet couplings," in IEEE Transactions on Magnetics, vol. 29, no. 6, pp. 2932-2934, Nov. 1993.

[2] W. Wu, H. C. Lovatt and J. B. Dunlop, "Analysis and design optimisation of magnetic couplings using 3D finite element modelling," in IEEE Transactions on Magnetics, vol. 33, no. 5, pp. 4083-4094, Sept. 1997.

[3] J. Kim, J. Choi, M. Koo, H. Shin and S. Lee, "Characteristic Analysis of Tubular-Type Permanent-Magnet Linear Magnetic Coupling Based on Analytical Magnetic Field Calculations," in IEEE Transactions on Applied Superconductivity, vol. 26, no. 4, pp. 1-5, June 2016.

[4] Radzevich, S. P., \& Dudley, D. W. (2012). Dudley's handbook of practical gear design and manufacture. Boca Raton, Fla: CRC Press.

[5] A. Al-Timimy et al., "Design and optimization of a high power density machine for flooded industrial pump," 2016 XXII International Conference on Electrical Machines (ICEM), Lausanne, 2016, pp. 1480-1486

[6] M. Lukic, P. Giangrande, A. Hebala, S. Nuzzo and M. Galea, "Review, Challenges, and Future Developments of Electric Taxiing Systems," in IEEE Transactions on Transportation Electrification, vol. 5, no. 4, pp. 1441-1457, Dec. 2019

[7] H. Zhang et al., "Thermal Model Approach to Multisector ThreePhase Electrical Machines," in IEEE Transactions on Industrial Electronics, pp. 1-1, 2020.

[8] P. Giangrande et al., "Considerations on the Development of an Electric Drive for a Secondary Flight Control Electromechanical Actuator," in IEEE Transactions on Industry Applications, vol. 55, no. 4, pp. 3544-3554, July-Aug. 2019.

[9] A. Al-Timimy, P. Giangrande, M. Degano, M. Galea and C. Gerada, "Comparative study of permanent magnet-synchronous and permanent magnet-flux switching machines for high torque to inertia applications," 2017 IEEE Workshop on Electrical Machines Design, Control and Diagnosis (WEMDCD), Nottingham, 2017, pp. 45-51.

[10] S. A. Odhano, P. Giangrande, R. Bojoi and C. Gerada, "Selfcommissioning of interior permanent magnet synchronous motor drives with high-frequency current injection," 2013 IEEE Energy Conversion Congress and Exposition, Denver, CO, 2013, pp. 38523859.

[11] P. Giangrande, F. Cupertino and G. Pellegrino, "Modelling of linear motor end-effects for saliency based sensorless control," 2010 IEEE Energy Conversion Congress and Exposition, Atlanta, GA, 2010, pp. 3261-3268.
[12] M. Galea, P. Giangrande, V. Madonna and G. Buticchi, "ReliabilityOriented Design of Electrical Machines: The Design Process for Machines' Insulation Systems MUST Evolve," in IEEE Industrial Electronics Magazine, vol. 14, no. 1, pp. 20-28, March 2020

[13] Z. Huang, T. Yang, P. Giangrande, S. Chowdhury, M. Galea and P. Wheeler, "An Active Modulation Scheme to Boost Voltage Utilization of the Dual Converter With a Floating Bridge," in IEEE Transactions on Industrial Electronics, vol. 66, no. 7, pp. 5623-5633, July 2019

[14] P. Giangrande, V. Madonna, S. Nuzzo and M. Galea, "Moving Toward a Reliability-Oriented Design Approach of Low-Voltage Electrical Machines by Including Insulation Thermal Aging Considerations," in IEEE Transactions on Transportation Electrification, vol. 6, no. 1, pp. 16-27, March 2020

[15] T. Lubin, S. Mezani and A. Rezzoug, "Simple Analytical Expressions for the Force and Torque of Axial Magnetic Couplings," in IEEE Transactions on Energy Conversion, vol. 27, no. 2, pp. 536-546, June 2012.

[16] H. Kang, J. Choi, H. Cho and J. Kim, "Comparative Study of Torque Analysis for Synchronous Permanent Magnet Coupling With Parallel and Halbach Magnetized Magnets Based on Analytical Field Calculations," in IEEE Transactions on Magnetics, vol. 50, no. 11, pp. 1-4, Nov. 2014.

[17] A. Al-Timimy, G. Vakil, M. Degano, P. Giangrande, C. Gerada and M. Galea, "Considerations on the Effects That Core Material Machining Has on an Electrical Machine's Performance," in IEEE Transactions on Energy Conversion, vol. 33, no. 3, pp. 1154-1163, Sept. 2018.

[18] V. Lemarquand, J. F. Charpentier and G. Lemarquand, "Nonsinusoidal torque of permanent-magnet couplings," in IEEE Transactions on Magnetics, vol. 35, no. 5, pp. 4200-4205, Sept. 1999.

[19] T. Lubin, S. Mezani and A. Rezzoug, "Steady-state and transient analysis of an axial-field magnetic coupling," 2012 XXth International Conference on Electrical Machines, Marseille, 2012.

[20] P. Giangrande, C. I. Hill, S. V. Bozhko and C. Gerada, "A novel multi-level electro-mechanical actuator virtual testing and analysis tool," 7th IET International Conference on Power Electronics, Machines and Drives (PEMD 2014), Manchester, 2014, pp. 1-6.

[21] Y. Akcay, P. Giangrande, M. Galea, C. Gerada, "Comparative Analysis between Axial and Coaxial Magnetic Couplings," accepted for publication in 10th IET International Conference on Power Electronics, Machines and Drives, 2020. 